\title{
Analogs of the $m$-function in the theory of orthogonal polynomials on the unit circle
}

\author{
Barry Simon ${ }^{1}$ \\ Department of Mathematics 253-37, California Institute of Technology, Pasadena, CA 91125, USA
}

Received 1 October 2003; accepted 19 January 2004

Dedicated to Norrie Everitt, on his 80th birthday, a bouquet to the master of the $m$-function

\begin{abstract}
We show that the multitude of applications of the Weyl-Titchmarsh $m$-function leads to a multitude of different functions in the theory of orthogonal polynomials on the unit circle that serve as analogs of the $m$-function.

(c) 2004 Elsevier B.V. All rights reserved.
\end{abstract}

Keywords: $m$-function; Orthogonal polynomials; Verblunsky coefficients

\section{Introduction}

Use of the Weyl-Titchmarsh $m$-function has been a constant theme in Norrie Everitt's opus, so I decided a discussion of the analogs of these ideas in the theory of orthogonal polynomials on the unit circle (OPUC) was appropriate. Interestingly enough, the uses of the $m$-functions are so numerous that OPUC has multiple analogs of the $m$-function!

$m$-functions are associated to solutions of

$$
-u^{\prime \prime}+q u=z u
$$

with $q$ a real function on $[0, \infty)$ and $z$ a parameter in $\mathbb{C}_{+}=\{z \mid \operatorname{Im} z>0\}$. The most fundamental aspect of the $m$-function is its relation to the spectral measure, $\rho$, for (1.1) by

$$
m(z)=c+\int \mathrm{d} \rho(x)\left[\frac{1}{x-z}-\frac{x}{1+x^{2}}\right],
$$

\footnotetext{
${ }^{1}$ Supported in part by NSF Grant DMS-0140592. For special issue of J. Comp. Appl. Math.

E-mail address: bsimon@caltech.edu (B. Simon).
} 
where $c$ is determined by (see $[3,13])$ :

$$
m(z)=\sqrt{-z}+\mathrm{o}(1) \quad \text { as } z \rightarrow \mathrm{i} \infty .
$$

Eqs. (1.2) plus (1.3) allow you to compute $m$ given $\mathrm{d} \rho$, and $\mathrm{d} \rho$ is determined by $m$ via

$$
\lim _{e \downarrow 0} \frac{1}{\pi} \int_{a}^{b} m(x+\mathrm{i} \varepsilon) \mathrm{d} x=\frac{1}{2}[\rho((a, b))+\rho([a, b])] .
$$

Of course, I have not told you what $m$ or $\rho$ is. This is done by defining $m$, in which case $\rho$ is defined by (1.4). Under weak conditions on $q$ at $\infty$, for $z \in \mathbb{C}_{+},(1.1)$ has a solution $u(x, z)$ which is $L^{2}$ at infinity, and it is unique up to a constant multiple. Then, $m$ is defined by

$$
m(z)=\frac{u^{\prime}(0, z)}{u(0, z)} .
$$

With this definition, $\mathrm{d} \rho$ is a spectral measure for $u \mapsto-u^{\prime \prime}+q u=H u$ in the sense that $H$ is unitarily equivalent to multiplication by $\lambda$ on $L^{2}(\mathbb{R}, \mathrm{d} \rho)$. (1.5) is often written in the equivalent form,

$$
\psi(x, z)+m(z) \varphi(x, z) \in L^{2},
$$

where $\varphi, \psi$ solve $(1.1)$ with initial conditions $\varphi(0)=0, \varphi^{\prime}(0)=1, \psi(0)=1, \psi^{\prime}(0)=0$.

Note that if one defines

$$
m(x ; z)=\frac{u^{\prime}(x, z)}{u(x, z)},
$$

the $m$-function for $q_{x}(\cdot)=q(\cdot+x)$, then $m$ obeys the Riccati equation

$$
m^{\prime}=q-z-m^{2} .
$$

It could be said that this is backwards: definition (1.5) should come first, before (1.2). I put it in this order because it is (1.2) that makes $m$ such an important object both in classical results $[2,5,7-9,16,23,33]$ and very recent work [4,10,21,25,27,31].

To describe the third role of the $m$-function, it will pay to switch to the case of Jacobi matrices. We now have, instead of $q$, two sequences $\left\{a_{n}\right\}_{n=1}^{\infty},\left\{b_{n}\right\}_{n=1}^{\infty}$ with $a_{n}>0, b_{n} \in \mathbb{R}$ which we will suppose uniformly bounded. Define an infinite matrix

$$
J=\left(\begin{array}{ccccc}
b_{1} & a_{1} & 0 & 0 & \cdots \\
a_{1} & b_{2} & a_{2} & 0 & \cdots \\
0 & a_{2} & b_{3} & a_{3} & \ldots \\
\vdots & \vdots & \vdots & \vdots & \ddots
\end{array}\right)
$$

which is a bounded self-adjoint operator. One defines

$$
m(z)=\left\langle\delta_{1},(J-z)^{-1} \delta_{1}\right\rangle .
$$

In terms of the spectral measure, $\mu$, for $\delta_{1}$ for $J$,

$$
m(z)=\int \frac{\mathrm{d} \mu(x)}{x-z} .
$$


If $u_{n}$ is the $\ell^{2}$ solution of $a_{n-1} u_{n-1}+\left(b_{n-z}\right) u_{n}+a_{n} u_{n+1}=0$ with $\operatorname{Im} z>0$, one has the analog of $(1.5)$

$$
m(z)=\frac{u_{1}(z)}{u_{0}(z)} .
$$

This process of going from $a$ and $b$ to $m$ and then to $\mu$ can be reversed. One way is by iterating (1.5) below, which lets one go from $\mu$ to $m$ (by (1.10)) and then gets the $a$ 's and $b$ 's as coefficients in a continued fraction expansion of $m$. From our point of view, an even more important way of going backwards uses orthogonal polynomials on the real line (OPRL). Given $\mu$ (of bounded support), one forms the monic orthogonal polynomials $P_{n}(x)$ for $\mathrm{d} \mu$ and shows they obey a recursion relation

$$
P_{n+1}(x)=\left(x-b_{n+1}\right) P_{n}(x)-a_{n}^{2} P_{n-1}(x)
$$

which yields the Jacobi parameters $a$ and $b$. The orthonormal polynomials, $p_{n}(x)$, are related to $P_{n}$ by

$$
p_{n}(x)=\left(a_{1} \ldots a_{n}\right)^{-1} P_{n}(x)
$$

and obey

$$
a_{n+1} p_{n+1}(x)=\left(x-b_{n+1}\right) p_{n}(x)-a_{n} p_{n-1}(x) .
$$

Eq. (1.7) has the analog

$$
m(z ; J)=\left(b_{1}-z-a_{1}^{2} m\left(z ; J^{(1)}\right)\right)^{-1},
$$

where $J^{(1)}$ is the Jacobi matrix with parameters $\tilde{a}_{m}=a_{m+1} \tilde{b}_{m}=b_{m+1}$ (i.e., the top row and left column are removed).

If $m(x+\mathrm{i} \varepsilon ; J)$ has a limit as $\varepsilon \downarrow 0,(1.15)$ says that $m\left(x+\mathrm{i} \varepsilon ; J^{(1)}\right)$ has a limit, and by (1.15),

$$
\frac{\operatorname{Im} m(x ; J)}{\operatorname{Im} m\left(x ; J^{(1)}\right)}=\left|a_{1} m(x ; J)\right|^{2} \text {. }
$$

$\operatorname{Im} m$ is important because if $\mu$ is given by (1.10), then

$$
\mathrm{d} \mu_{\mathrm{ac}}=\frac{1}{\pi} \operatorname{Im} m(x+\mathrm{i} 0) \mathrm{d} x .
$$

This property of $m$, that its energy is the ratio of Im's, is a critical element of recent work on sum rules for spectral theory $[6,19,28-30]$.

The interesting point is that, for OPUC, the analogs of the functions obeying (1.2), (1.5), and (1.16) are different! In Section 2, we will give a quick summary of OPUC. In Section 3, we discuss (1.2); in Section 4, we discuss (1.16); and finally, in Section 5, the analog of (1.5).

Happy 80th, Norrie. I hope you enjoy this bouquet.

\section{Overview of OPUC}

We want to discuss here the basics of OPUC, although we will only scratch the surface of a rich and beautiful subject [29]. The theory reverses the usual passage from differential/difference equations to measures, and instead follows the discussion of OPRL in Section 1. $\mu$ is now a probability measure on $\partial \mathbb{D}=\{z|| z \mid=1\}$. We suppose $\mu$ is nontrivial, that is, not supported on a finite set. One can then 
form, by the Gram-Schmidt procedure, the monic orthogonal polynomials $\Phi_{n}(z)$ and the orthonormal polynomials, $\varphi_{n}(z)=\Phi_{n}(z) /\left\|\Phi_{n}\right\|$ where $\|\cdot\|$ is the $L^{2}(\partial \mathbb{D}, \mathrm{d} \mu)$ norm.

Given fixed $n \in\{0,1,2, \ldots\}$, we define an anti-unitary operator on $L^{2}(\partial \mathbb{D}, \mathrm{d} \mu)$ by

$$
f^{*}(z)=z^{n} \overline{f(z)} \text {. }
$$

The use of a symbol without " $n$ " is terrible notation, but it is standard! If $Q_{n}$ is a polynomial of degree $n, Q_{n}^{*}$ is also a polynomial of degree $n$. Indeed,

$$
Q_{n}^{*}(z)=z^{n} \overline{Q_{n}(1 / \bar{z})}
$$

so if $Q_{n}(z)=a_{n} z^{n}+a_{n-1} z^{n-1}+\cdots+a_{0}$, then $Q_{n}^{*}(z)=\bar{a}_{0} z^{n}+\bar{a}_{1} z^{n-1}+\cdots+\bar{a}_{n}$.

Since $\Phi_{n}$ is monic, $\Phi_{n}^{*}(0)=1$, and thus, $N(z) \equiv\left(\Phi_{n+1}^{*}(z)-\Phi_{n}^{*}(z)\right) / z$ is a polynomial of degree $n$. Since ${ }^{*}$ is anti-unitary,

$$
\begin{aligned}
\left\langle z^{m}, N(z)\right\rangle & =\left\langle z^{m+1}, \Phi_{n+1}^{*}-\Phi_{n}^{*}\right\rangle \\
& =\left\langle\Phi_{n+1}, z^{n+1-(m+1)}\right\rangle-\left\langle\Phi_{n}, z^{n-m-1}\right\rangle \\
& =0,
\end{aligned}
$$

for $m=0,1, \ldots, n-1$. Thus $N(z)$ must be a multiple of $\Phi_{n}(z)$, that is, for some $\alpha_{n} \in \mathbb{C}$,

$$
\Phi_{n+1}^{*}(z)=\Phi_{n}^{*}(z)-\alpha_{n} z \Phi_{n}(z)
$$

and its ${ }^{*}$,

$$
\Phi_{n+1}(z)={ }_{z} \Phi_{n}(z)-\bar{\alpha}_{n} \Phi_{n}^{*}(z) .
$$

$(2.2) /(2.3)$ are the Szegö recursion formulae ([32]); the $\alpha_{n}$ 's are the Verblunsky coefficients (after [34]). The derivation I have just given is that of Atkinson [2].

Since $\Phi_{n}^{*} \perp \Phi_{n+1}$, (2.3) implies

$$
\left\|\Phi_{n+1}\right\|^{2}+\left|\alpha_{n}\right|^{2}\left\|\Phi_{n}^{*}\right\|^{2}=\left\|z \Phi_{n}\right\|^{2} .
$$

Since $\left\|\Phi_{n}^{*}\right\|=\left\|z \Phi_{n}\right\|=\left\|\Phi_{n}\right\|$, we have

$$
\left\|\Phi_{n+1}\right\|=\left(1-\left|\alpha_{n}\right|^{2}\right)^{1 / 2}\left\|\Phi_{n}\right\| .
$$

This implies first of all that

$$
\left|\alpha_{n}\right|<1
$$

and if

$$
\rho_{n} \equiv\left(1-\left|\alpha_{n}\right|^{2}\right)^{1 / 2},
$$

then

$$
\|\Phi\|_{n}=\rho_{0} \rho_{1} \ldots \rho_{n-1}
$$

so

$$
\varphi_{n}=\left(\rho_{0} \ldots \rho_{n-1}\right)^{-1} \Phi_{n}
$$


and (2.2), (2.3) becomes

$$
\begin{aligned}
& z \varphi_{n}=\rho_{n} \varphi_{n+1}+\bar{\alpha}_{n} \varphi_{n}^{*}, \\
& \varphi_{n}^{*}=\rho_{n}, \varphi_{n+1}^{*}+\alpha_{n} z \varphi_{n} .
\end{aligned}
$$

The $\alpha_{n}$ 's not only lie in $\mathbb{D}$, but it is a theorem of Verblunsky [34] that as $\mu$ runs through all nontrivial measures, the set of $\alpha$ 's runs through all of $\times_{n=0}^{\infty} \mathbb{D}$. The $\alpha$ 's are the analogs of the $a$ 's and $b$ 's in the Jacobi case or of $V$ in the Schrödinger case.

We will later have reason to consider Szegö's theorem in Verblunsky's form [35].

Theorem 2.1. Let

$$
\mathrm{d} \mu=w \frac{\mathrm{d} \theta}{2 \pi}+\mathrm{d} \mu_{\mathrm{s}}
$$

Then

$$
\prod_{j=0}^{\infty}\left(1-\left|\alpha_{j}\right|^{2}\right)=\exp \left(\int \log (w(\theta)) \frac{\mathrm{d} \theta}{2 \pi}\right) .
$$

Remark. The log integral can diverge to $-\infty$. The theorem says the integral is $-\infty$ if and only if the product on the left is 0 , that is, if and only if $\sum\left|\alpha_{j}\right|^{2}=\infty$.

If

$$
\sum_{j=0}^{\infty}\left|\alpha_{j}\right|^{2}<\infty
$$

we say the Szegö condition holds. This happens if and only if

$$
\int|\log (w(\theta))| \frac{\mathrm{d} \theta}{2 \pi}<\infty .
$$

In that case, we define the Szegö function on $\mathbb{D}$ by

$$
D(z)=\exp \left(\int \frac{\mathrm{e}^{\mathrm{i} \theta}+z}{\mathrm{e}^{\mathrm{i} \theta}-z} \log (w(\theta)) \frac{\mathrm{d} \theta}{4 \pi}\right) .
$$

\section{The Carathéodory and Schur functions}

Given (1.10) (and (1.2)), the natural " $m$-function" for OPUC is the Carathéodory function, $F(z)$,

$$
F(z)=\int \frac{\mathrm{e}^{\mathrm{i} \theta}+z}{\mathrm{e}^{\mathrm{i} \theta}-z} \mathrm{~d} \mu(\theta) .
$$

The Cauchy kernel $\left(\mathrm{e}^{\mathrm{i} \theta}+z\right) /\left(\mathrm{e}^{\mathrm{i} \theta}-z\right)$ has the Poisson kernel

$$
\left.\operatorname{Re}\left(\frac{\mathrm{e}^{\mathrm{i} \theta}+z}{\mathrm{e}^{\mathrm{i} \theta}-z}\right)\right|_{z=r \mathrm{e}^{\mathrm{i} \varphi}}=\frac{1-r^{2}}{1+r^{2}-2 \cos (\theta-\varphi)}
$$


as its real part, and this is positive, so

$$
\operatorname{Re} F(z)>0 \quad \text { for } z \in \mathbb{D}, \quad F(0)=1 .
$$

This replaces $\operatorname{Im} m>0$ if $\operatorname{Im} z>0$.

One might think the "correct" analog of $m$ is

$$
R(z)=\int \frac{1}{\mathrm{e}^{\mathrm{i} \theta}-z} \mathrm{~d} \mu(\theta) .
$$

$R$ and $F$ are related by

$$
R(z)=(2 z)^{-1}(F(z)-1) .
$$

If one rotates $\mathrm{d} \mu$ and $z$ (i.e., $\left.\mathrm{d} \mu(\theta) \rightarrow \mathrm{d} \mu(\theta-\varphi), z \rightarrow \mathrm{e}^{\mathrm{i} \varphi} z\right), F$ is unchanged but $R$ is multiplied by $\mathrm{e}^{-\mathrm{i} \varphi}$, so the set of values $R$ can take are essentially arbitrary-which shows $F$, which obeys $\operatorname{Re} F(z)>0$, is a nicer object to take. That said, we will see $R$ again in Section 5 .

$F$ has some important analogs of $m$ :

(1) $\lim _{r \uparrow 1} F\left(r \mathrm{e}^{\mathrm{i} \theta}\right)$ exists for a.e. $\theta$, and if (2.11) defines $w$, then

$$
w(\theta)=\operatorname{Re} F\left(\mathrm{e}^{\mathrm{i} \theta}\right) .
$$

(2) $\theta_{0}$ is a pure point of $\mu$ if and only if $\lim _{r \uparrow 1}(1-r) \operatorname{Re} F\left(r \mathrm{e}^{\mathrm{i} \theta_{0}}\right) \neq 0$ and, in general,

$$
\lim _{r \uparrow 1}(1-r) \operatorname{Re} F\left(r \mathrm{e}^{\mathrm{i} \theta_{0}}\right)=\mu\left(\left\{\theta_{0}\right\}\right) .
$$

(3) $\mathrm{d} \mu_{\mathrm{s}}$ is supported on $\left\{\theta \mid \lim _{r \uparrow 1} F\left(r \mathrm{e}^{\mathrm{i} \theta}\right)=\infty\right\}$.

In fact, the proof of the analogs of these facts for $m$ proceeds by mapping $\mathbb{C}_{+}$to $\mathbb{D}$ and using these facts for $F$ !

These properties provide a strong analogy, but one can note a loss of "symmetry" relative to the ODE case. The $m$-function maps $\mathbb{C}_{+}$to $\mathbb{C}_{+} \cdot F$ though maps $\mathbb{D}$ to $-\mathrm{i} \mathbb{C}_{+}$. One might prefer a map of $\mathbb{D}$ to $\mathbb{D}$. In fact, one defines the Schur function, $f$, of $\mu$ via

$$
F(z)=\frac{1+z f(z)}{1-z f(z)},
$$

then $f$ maps $\mathbb{D}$ to $\mathbb{D}$ and (3.7) sets up a one-one correspondence between $F$ 's with $\operatorname{Re} F>0$ on $\mathbb{D}$ and $F(0)=1$ and $f$ mapping $\mathbb{D}$ to $\mathbb{D}$ (this fact relies on the Schwarz lemma that $f$ maps $\mathbb{D}$ to $\mathbb{D}$ with $f(0)=0$ if and only if $f=z g$ where $g$ maps $\mathbb{D}$ to $\mathbb{D}$ ).

For at least some purposes, $f$ is a "better" analog of $m$ than $F$, for example, in regard to its analog of the recursion (1.10). If $f$ is the Schur function associated to Verblunsky coefficients $\left\{\alpha_{0}, \alpha_{1}, \ldots\right\}$ and $f_{n}$ is the Schur function associated to $\left\{\alpha_{n}, \alpha_{n+1}, \ldots\right\}$, then

$$
f=\frac{\alpha_{0}+z f_{1}}{1+\bar{\alpha}_{0} z f_{1}},
$$

a result of Geronimus (see [29] for lots of proofs of this fact).

Interestingly enough, Schur, not knowing of the connection to OPUC, discussed (3.8) for $\alpha_{0}=f(0)$ as a map of $f \rightarrow\left(\alpha_{0}, f_{1}\right)$ and, by iteration, to a parametrization of functions of $\mathbb{D}$ to $\mathbb{D}$ by parameters 
$\alpha_{0}, \ldots, \alpha_{n}, \ldots$. There is, of course, a formula relating $F$ to $F_{1}$ that can be obtained from (3.7) and (3.8) or directly [22], but it is more complicated than (3.8).

Finally, in discussing $f$, we note that there is a natural family $\left\{\mathrm{d} \mu_{\lambda}\right\}_{\lambda \in \partial \mathbb{D}}$ of measures related to $\mathrm{d} \mu$ (with $\mathrm{d} \mu_{\lambda=1}=\mathrm{d} \mu$ ) that corresponds to "varying boundary conditions." We will discuss those more fully in Section 5 , but we note

$$
f\left(z ; \mathrm{d} \mu_{\lambda}\right)=\lambda f(z ; \mathrm{d} \mu)
$$

while the formula for $F\left(\mathrm{~d} \mu_{\lambda}\right)$ is more involved.

The Schur function and Schur iterates, $f_{n}$, have been used by Khrushchev $[14,17,18]$ as a powerful tool in the analysis of OPUC.

\section{The relative Szegö function}

As explained in the Introduction, a critical property of $m$ is (1.16), which is the basis of step-by-step sum rules (see [28]). The left side of (1.16) enters as the ratio of a.c. weights of $\mathrm{d} \mu_{J}$ and $\mathrm{d} \mu_{J^{(1)}}$. Thus, we are interested in $\operatorname{Im} F\left(\mathrm{e}^{\mathrm{i} \theta} ;\left\{\alpha_{j}\right\}_{j=0}^{\infty}\right)$ divided by $\operatorname{Im} F\left(\mathrm{e}^{\mathrm{i} \theta} ;\left\{\alpha_{j+1}\right\}_{j=0}^{\infty}\right)$, that is, $\operatorname{Im} F / \operatorname{Im} F_{1}$ in the language of the last section. Neither $|F|$ nor $|f|$ is directly related to this ratio, so we need a different object to get an analog of (1.16). The following was introduced by Simon in [29]:

$$
\left(\delta_{0} D\right)(z)=\frac{1-\bar{\alpha}_{0} f}{\rho_{0}} \frac{1-z f_{1}}{1-z f} .
$$

It is called the "relative Szego" function" for reasons that will become clear in a moment.

In (4.1), $f_{1}$ is the Schur function for Verblunsky coefficients

$$
\alpha_{j}^{(1)}=\alpha_{j+1} \text {. }
$$

Here is the key fact:

Theorem 4.1. Let $\mathrm{d} \mu$ and $\mathrm{d} \mu^{(1)}$ be measures on $\partial \mathbb{D}$ with Verblunsky coefficients related by (4.2). Suppose $\mathrm{d} \mu=w(\theta) \mathrm{d} \theta / 2 \pi+\mathrm{d} \mu_{\mathrm{s}}$ and $\mathrm{d} \mu^{(1)}=w^{(1)} \mathrm{d} \theta / 2 \pi+\mathrm{d} \mu_{\mathrm{s}}$. Then

(1) For a.e. $\theta, \lim _{r \uparrow 1}\left(\delta_{0} D\right)\left(r \mathrm{e}^{\mathrm{i} \theta}\right) \equiv \delta_{0} D\left(\mathrm{e}^{\mathrm{i} \theta}\right)$ exists.

(2) If $w(\theta) \neq 0$, then (for a.e. $\theta$ w.r.t. $\mathrm{d} \theta / 2 \pi), w_{1}(\theta) \neq 0$ and

$$
\frac{w(\theta)}{w_{1}(\theta)}=\left|\left(\delta_{0} D\right)\left(\mathrm{e}^{\mathrm{i} \theta}\right)\right|^{2} .
$$

Sketch of Proof. Each of the functions $1-\bar{\alpha}_{0} f, 1-z f_{1}$, and $1-z f$ takes values in $\{w|| w-1 \mid<1\}$ on $\mathbb{D}$, so their arguments lie in $[-\pi / 2, \pi / 2]$, so their logs are in all $H^{p}, 1<p<\infty$. That is, they are outer functions, and so $\delta_{0} D$ is an outer function, which means that assertion (1) holds (see Rudin [24] for a pedagogic discussion of outer functions).

To get (4.3), we note that (3.7) implies

$$
\operatorname{Re} F(z)=\frac{1-|f|^{2}|z|^{2}}{|1-z f|^{2}}
$$


SO

$$
\frac{\operatorname{Re} F(z)}{\operatorname{Re} F_{1}(z)}=\left|\frac{1-z f_{1}}{1-z f}\right|^{2} \frac{1-|f|^{2}|z|^{2}}{1-\left|f_{1}\right|^{2}|z|^{2}} .
$$

On the other hand, (3.8) implies

$$
z f_{1}=\frac{f-\alpha_{0}}{1-\bar{\alpha}_{0} f},
$$

which implies

$$
1-\left|z f_{1}\right|^{2}=\frac{\rho_{0}^{2}\left(1-|f|^{2}\right)}{\left|1-\bar{\alpha}_{0} f\right|^{2}}
$$

so, putting these formulae together,

$$
\frac{\operatorname{Re} F(z)}{\operatorname{Re} F_{1}(z)}=\left|\left(\delta_{0} D\right)(z)\right|^{2}\left(\frac{1-|z|^{2}|f|^{2}}{1-|f|^{2}}\right)
$$

which, as $|z| \rightarrow 1$, yields (4.3).

In particular, one has the nonlocal step-by-step sum rule that if $w(\theta) \neq 0$ for a.e. $\theta$, then

$$
\left(\delta_{0} D\right)(z)=\exp \left(\int_{0}^{2 \pi} \frac{\mathrm{e}^{\mathrm{i} \theta}+z}{\mathrm{e}^{\mathrm{i} \theta}-z} \log \left(\frac{w(\theta)}{w_{1}(\theta)}\right) \frac{\mathrm{d} \theta}{4 \pi}\right)
$$

and, in particular, setting $z=0$,

$$
\rho_{0}^{2}=\exp \left(\int_{0}^{2 \pi} \log \left(\frac{w(\theta)}{w_{1}(\theta)}\right) \frac{\mathrm{d} \theta}{2 \pi}\right)
$$

which is not only consistent with Szegö's theorem (2.11) but, using semicontinuity of the entropy, can be used to prove it (see $[19,29]$ ) as follows:

(1) Iterating (4.9) yields

$$
\left(\rho_{0} \ldots \rho_{n-1}\right)^{2}=\exp \left(\int_{0}^{2 \pi} \log \left(\frac{w(\theta)}{w_{n}(\theta)}\right) \frac{\mathrm{d} \theta}{2 \pi}\right) .
$$

(2) Since $\exp \left(\int_{0}^{2 \pi} \log \left(w_{n}(\theta) \mathrm{d} \theta / 2 \pi\right) \leqslant \int_{0}^{2 \pi} w_{n}(\theta) \mathrm{d} \theta / 2 \pi \leqslant 1\right.$, (4.10) implies

$$
\left(\rho_{0} \ldots \rho_{n-1}\right)^{2} \geqslant \exp \left(\int_{0}^{2 \pi} \log (w(\theta)) \frac{\mathrm{d} \theta}{2 \pi}\right) .
$$

(3) If $w^{(n)}$ is the weight associated to the measure with

$$
\alpha_{j}^{(n)}= \begin{cases}\alpha_{j}, & j \leqslant n-1 \\ 0, & j \geqslant n,\end{cases}
$$

(4.10) proves

$$
\left(\rho_{0} \ldots \rho_{n-1}\right)^{2}=\exp \int_{0}^{2 \pi} \log \left(w^{(n)}(\theta)\right) \frac{\mathrm{d} \theta}{2 \pi} .
$$


(4) $\mathrm{d} \mu \rightarrow \int_{0}^{2 \pi} \log (w(\theta)) \mathrm{d} \theta / 2 \pi$ is an entropy, hence, weakly upper semicontinuous. Since $w^{(n)} \mathrm{d} \theta /$ $2 \pi \rightarrow \mathrm{d} \mu$ weakly as $n \rightarrow \infty$, this semicontinuity shows

$$
\lim _{n \rightarrow \infty}\left(\rho_{n} \ldots \rho_{n-1}\right)^{2} \leqslant \exp \left(\int_{0}^{2 \pi} \log (w(\theta)) \frac{\mathrm{d} \theta}{2 \pi}\right) .
$$

Eqs. (4.11) and (4.13) is Szegö's theorem.

Two other properties of $\delta_{0} D$ that we should mention are:

(A) If $\sum_{n=0}^{\infty}\left|\alpha_{n}\right|^{2}<\infty$, then

$$
\left(\delta_{0} D\right)(z)=\frac{D\left(z ; \alpha_{0}, \alpha_{1}, \alpha_{2}, \ldots\right)}{D\left(z ; \alpha_{1}, \alpha_{2}, \alpha_{3}, \ldots\right)} .
$$

(B) In general, one has

$$
\delta_{0} D(z)=\lim _{n \rightarrow \infty} \frac{\varphi_{n-1}^{*}\left(z ; \alpha_{1}, \alpha_{2}, \ldots\right)}{\varphi_{n}^{*}\left(z ; \alpha_{0}, \alpha_{1}, \ldots\right)} .
$$

\section{Eigenfunction ratios}

Finally, we look at the analogs of $m$ as a function ratio, its initial definition by Weyl and Titchmarsh. The key papers on this point of view are by Geronimo-Teplyaev [11] and Golinskii-Nevai [15]. We will see from one point of view [15] that $F(z)$ plays this role, but from other points of view [11] that other functions are more natural.

The recursion relations $(2.9) /(2.10)$ can be rewritten as

$$
\left(\begin{array}{c}
\varphi_{n+1} \\
\varphi_{n+1}^{*}
\end{array}\right)=A\left(\alpha_{n}, z\right)\left(\begin{array}{c}
\varphi_{n} \\
\varphi_{n}^{*}
\end{array}\right),
$$

where

$$
A(\alpha, z)=\rho^{-1}\left(\begin{array}{cc}
z & -\bar{\alpha}_{n} \\
-\alpha_{n} z & 1
\end{array}\right)
$$

(with $\left.\rho=\left(1-\mid \alpha^{2}\right)^{1 / 2}\right)$. From this point of view, the analog of the fundamental differential/difference equation in the real case is

$$
\Xi_{n}=T_{n}(z) \Xi_{0}
$$

with

$$
T_{n}(z)=A\left(\alpha_{n-1}, z\right) \cdots A\left(\alpha_{0}, z\right) .
$$

The correct boundary conditions for the usual OPUC are $\Xi_{0}=\left(\begin{array}{l}1 \\ 1\end{array}\right)$.

One can ask for what other initial conditions the polynomials associated with the top component of $T_{n}(z) \Xi_{0}$ are OPUC for some measure. Note that

$$
\left(\begin{array}{l}
1 \\
\lambda
\end{array}\right)=U(\lambda)\left(\begin{array}{l}
1 \\
1
\end{array}\right)
$$


with

$$
U(\lambda)=\left(\begin{array}{ll}
1 & 0 \\
0 & \lambda
\end{array}\right)
$$

and that

$$
U(\lambda)^{-1} A(\alpha, z) U(\lambda)=\rho^{-1}\left(\begin{array}{cc}
z & -\bar{\alpha}_{n} \lambda \\
-\alpha_{n} \lambda^{-1} z & 1
\end{array}\right) .
$$

We see from this that $\bar{\lambda}=\lambda^{-1}$, that is, $|\lambda|=1$ will yield $U(\lambda)^{-1} A\left(\alpha_{1}, z\right) U(\lambda)=A(\bar{\lambda} \alpha, z)$. Changing $\lambda$ to $\bar{\lambda}$, we see that

Proposition 5.1. Let $|\lambda|=1$. If $\varphi_{n}^{(\lambda)}(z)$ are the OPUC for Verblunsky coefficients $\alpha_{n}^{(\lambda)}=\lambda \alpha_{n}$, then

$$
\left(\begin{array}{c}
\varphi_{n}^{(\lambda)}(z) \\
\bar{\lambda} \varphi_{n}^{(\lambda) *}(z)
\end{array}\right)=T_{n}\left(z ;\left\{\alpha_{j}\right\}_{j=1}^{\infty}\right)\left(\begin{array}{c}
1 \\
\bar{\lambda}
\end{array}\right) .
$$

This suggests that one look at the family $\mathrm{d} \mu_{\lambda}$ or measures with

$$
\alpha_{j}\left(\mathrm{~d} \mu_{\lambda}\right)=\lambda \alpha_{j}(\mathrm{~d} \mu)
$$

called the family of Aleksandrov measures associated to $\left\{\alpha_{j}\right\}_{j=0}^{\infty}$ after [1]. The special case $\lambda=-1$ goes back to Verblunsky [35] and Geronimus [12], and are called the second kind polynomials, denoted $\psi_{n}(z)$. The following goes back to Verblunsky [35].

Theorem 5.2. For $z \in \mathbb{D}$, uniformly on compact subsets of $\mathbb{D}$,

$$
\lim _{n \rightarrow \infty} \frac{\psi_{n}^{*}(z)}{\varphi_{n}^{*}(z)}=F(z)
$$

Clearly related to this is the following result of Golinskii-Nevai [15]:

Theorem 5.3. Let $z \in \mathbb{D}$. Then

$$
\sum_{n=0}^{\infty}\left|\left(\begin{array}{c}
\psi_{n}(z) \\
-\psi_{n}^{*}(z)
\end{array}\right)+\beta\left(\begin{array}{c}
\varphi_{n}(z) \\
\varphi_{n}^{*}(z)
\end{array}\right)\right|^{2}<\infty
$$

if and only if

$$
\beta=F(z) \text {. }
$$

From this point of view, $F$ is again the "correct" analog of $m$ ! Indeed, the Golinskii-Nevai [15] proof uses Weyl limiting circles to prove the theorem (one is always in limit point case!).

But this is not the end of the story. Define

$$
u_{k}=\psi_{k}+F(z) \varphi_{k}, \quad u_{k}^{*}=-\psi_{k}^{*}+F(z) \varphi_{k}^{*}
$$


so $\left(\begin{array}{l}u_{k} \\ u_{k}^{*}\end{array}\right)$ is the unique solution of $\Xi_{n}=T_{n}(z) \Xi_{0}$ which is in $\ell^{2}$. In the OPRL case, the basic vector solution is of the form $\left(\begin{array}{c}u_{n} \\ u_{n+1}\end{array}\right)$, so we have the analog of (1.11),

$$
\tilde{m}(z)=\frac{u_{0}^{*}}{u_{0}}=\frac{-1+F}{1+F}=z f .
$$

So one analog of the $m$-function is $z f$.

In particular, (5.14) implies

$$
\left|u_{k}^{*}\right|<\left|u_{k}\right|
$$

for $z \in \mathbb{D}$, and thus the rate of exponential decay of $\left|\left(\begin{array}{c}u_{k} \\ u_{k}^{*}\end{array}\right)\right|$ is that of $u_{k}$. If there is such exponential decay in the sense that

$$
\gamma_{2}=\lim _{n \rightarrow \infty}\left[\left\|\left(\begin{array}{l}
u_{n} \\
u_{n}^{*}
\end{array}\right)\right\|^{1 / n}\right]
$$

exists, then, by (5.15),

$$
\gamma_{2}=\lim _{n \rightarrow \infty} \frac{1}{n} \sum_{j=0}^{n-1} \log \left|m_{n}^{+}\right|,
$$

where

$$
m_{n}^{+}=\frac{u_{n+1}}{u_{n}} .
$$

For $n=0, u_{1}=\psi_{1}+F \varphi_{1}, u_{0}=1+F, \psi_{1}=\rho_{0}^{-1}\left(z+\bar{\alpha}_{0}\right), \varphi_{1}=\rho_{0}^{-1}\left(z-\bar{\alpha}_{0}\right)$, so by a direct calculation,

$$
m_{0}^{+}(z)=\rho_{0}^{-1} z\left(1-\bar{\alpha}_{0} f\right),
$$

yet another reasonable choice for an $m$-function.

Indeed, if $\gamma(z)=\lim _{n \rightarrow \infty}(1 / n) \log \left\|T_{n}(z)\right\|$ exists, the fact that det $\left(T_{n}\right)=z^{n}$ implies that $\gamma=\log |\lambda|-$ $\gamma_{2}$, and one finds in the case of stochastic Verblunsky coefficients that $[11,29]$

$$
\mathbb{E}\left(\log \left|m_{\omega}^{+}(z)\right|\right)=\log |z|-\gamma(z),
$$

an analog of a fundamental formula of Kotani [20,26] that in his case uses $m$ !

Finally, we turn to the connection of $m$ to whole-line Green's functions. Given $V$ on $(-\infty, \infty)$ and $z \in \mathbb{C}_{+}$, it is natural to look at the two solutions of $(1.1), u_{ \pm}(x, z)$, which are $\ell^{2}$ on $\pm(0, \infty)$ and the $m$-functions,

$$
m_{ \pm}(z)= \pm \frac{u_{ \pm}^{\prime}(0, z)}{u_{ \pm}(0, z)}
$$

$m_{ \pm}$are the $m$-functions for $V( \pm x)[0, \infty)$. Standard Green's function formulae show that the integral kernel, $G(x, y ; z)$, of $\left(-\mathrm{d}^{2} / \mathrm{d} x^{2}+V-z\right)^{-1}$ is

$$
G(x, y ; z)=\frac{u_{-}\left(x_{<}\right) u_{+}\left(x_{>}\right)}{\left(u_{+}(0) u_{-}^{\prime}(0)-u_{+}^{\prime}(0) u_{-}(0)\right)},
$$

where $x_{<}=\min (x, y)$ and $x_{>}=\max (x, y)$. In particular,

$$
G(0,0 ; z)=-\left(m_{+}(z)+m_{-}(z)\right)^{-1} \text {. }
$$


A complete description of the OPUC analog would require too much space, so we sketch the ideas, leaving the details to [29]. Just as the difference equation is associated to a tridiagonal selfadjoint matrix whose spectral measure is the one generating the OPRL, any set of $\alpha$ 's is associated to a five-diagonal unitary matrix, called the CMV matrix, whose spectral measure is the $\mathrm{d} \mu$ with $\alpha_{j}(\mathrm{~d} \mu)=\alpha_{j}$.

The CMV matrix is one-sided, but given $\left\{\alpha_{j}\right\}_{j=-\infty}^{\infty}$, one can define a two-sided CMV matrix, $\mathscr{E}$, in a natural way. If $G(z)$ is the 00 matrix element of $(\mathscr{E}-z)^{-1}$, then (see $[11,17,29]$ )

$$
G(z)=\frac{f_{+}(z) f_{-}(z)}{1-z f_{+}(z) f_{-}(z)},
$$

where $f_{+}$is the Schur function for $\left(\alpha_{0}, \alpha_{1}, \alpha_{2}, \ldots\right)$ and $f_{-}$the Schur function for $\left(-\bar{\alpha}_{-1},-\bar{\alpha}_{-2}, \ldots\right)$. On the basis of the analogy between (5.23) and (5.22), Geronimo-Teplyaev [11] called $f_{+}$and $z f_{-}$ the $m_{+}$and $m_{-}$functions.

\section{Summary}

We have thus seen that there are many analogs of the $m$-function in the theory of OPUC:

(1) The Carathéodory function, $F(z)$, given by (3.1), an analog of (1.2) and also related to the classic Weyl definition $(5.11) /(5.12)$.

(2) The Schur function, $f(z)$, given by (3.7) with a recursion, (3.8), closer to the recursion (1.15) for the $m$-function of OPRL. $f$ also enters via (5.23).

(3) $z f(z)$, the $\tilde{m}$-function of (5.14).

(4) The relative Szegö function, (4.1), which, via (4.3) and (1.16), is an analog of $a_{1} m(z)$.

(5) The $m^{+}$-function, (5.19), which plays the role that $m$ does in Kotani theory.

\section{Note added in proof}

After this paper was processed, while finishing up the preparation of [29], I realized there is yet another OPUC analog of the $m$-function. A key property of the $m$-function for the Jacobi case is that $m$ has poles at eigenvalues of $J$ and zeros at eigenvalues of the Jacobi matrix obtained by removing one $a$ and one $b$. An analogous function for OPUC is

$$
M(z)=z\left(1+\alpha_{0}\right)(1+F(z))+\left(\bar{\alpha}_{0}+1\right)(1-F(z)) .
$$

This has poles at poles of $F$ and zeros at point masses for $\mathrm{d} \mu_{1}$, the measure associated to $\left\{\alpha_{j+1}\right\}_{j=0}^{\infty}$. There are two exceptions to this statement. It can happen at $z=\left(1+\bar{\alpha}_{0}\right) /\left(1+\alpha_{0}\right)$ that both measures have a pure point, in which case $M$ has neither a zero nor a pole (this kind of cancellation does not happen for Jacobi matrices because of interlacing of zeros). $M$ vanishes at $z=0$. This $M$-function continued to a hyperelliptic Riemann surface is critical to the analysis of finite gap Verblunsky coefficients; see [29]. 


\section{References}

[1] A.B. Aleksandrov, Multiplicity of boundary values of inner functions, Izv. Akad. Nauk Arm. SSR 22 (1987) 490-503.

[2] F.V. Atkinson, Discrete and Continuous Boundary Problems, Academic Press, New York, 1964.

[3] F.V. Atkinson, On the location of the Weyl circles, Proc. Roy. Soc. Edinburgh Sect. A 88 (1981) 345-356.

[4] G. Borg, Uniqueness theorems in the spectral theory of $y^{\prime \prime}+(\lambda-q(x)) y=0$, Proceedings of the 11 th Scandinavian Congress of Mathematicians, Trondheim, 1949, Johan Grundt Tanums Forlag, Oslo, 1952, pp. 276-287.

[5] E.A. Coddington, N. Levinson, Theory of Ordinary Differential Equations, McGraw-Hill, New York, 1955.

[6] D. Damanik, B. Simon, Jost functions and Jost solutions for Jacobi matrices, I. A necessary and sufficient condition for Szegö asymptotics, in preparation.

[7] M.S.P. Eastham, The Spectral Theory of Periodic Differential Equations, Scottish Academic Press, Edinburgh, 1973.

[8] M.S.P Eastham, Antibound states and exponentially decaying Sturm-Liouville potentials, J. London Math. Soc. 65 (2) (2002) 624-638.

[9] W.N. Everitt, M.K. Kwong, A. Zettl, Oscillation of eigenfunctions of weighted regular Sturm-Liouville problems, J. London Math. Soc. 27 (2) (1983) 106-120.

[10] I.M. Gel'fand, private communication.

[11] J.S. Geronimo, A. Teplyaev, A difference equation arising from the trigonometric moment problem having random reflection coefficients - an operator-theoretic approach, J. Funct. Anal. 123 (1994) 12-45.

[12] J. Geronimus, On the trigonometric moment problem, Ann. Math. 47 (2) (1946) 742-761.

[13] F. Gesztesy, B. Simon, A new approach to inverse spectral theory, II. General real potentials and the connection to the spectral measure, Ann. Math. 152 (2000) 593-643.

[14] L. Golinskii, S. Khrushchev, Cesàro asymptotics for orthogonal polynomials on the unit circle and classes of measures, J. Approx. Theory 115 (2002) 187-237.

[15] L. Golinskii, P. Nevai, Szegö difference equations, transfer matrices and orthogonal polynomials on the unit circle, Comm. Math. Phys. 223 (2001) 223-259.

[16] P. Hartman, Ordinary Differential Equations, Wiley, New York, 1964.

[17] S. Khrushchev, Schur's algorithm, orthogonal polynomials, and convergence of Wall's continued fractions in $L^{2}$ ( $\mathbb{T}$ ), J. Approx. Theory 108 (2001) 161-248.

[18] S. Khrushchev, Classification theorems for general orthogonal polynomials on the unit circle, J. Approx. Theory 116 (2002) 268-342.

[19] R. Killip, B. Simon, Sum rules for Jacobi matrices and their applications to spectral theory, Ann. Math. 158 (2) (2003) 253-321.

[20] S. Kotani, Lyapunov indices determine absolutely continuous spectra of stationary random one-dimensional Schrödinger operators, Stochastic Analysis, Katata/Kyoto, 1982, North-Holland Math. Library, 32, North-Holland, Amsterdam, 1984, pp. 225-247.

[21] B.M. Levitan, Inverse Sturm-Liouville Problems, VNU Science Press, Utrecht, 1987.

[22] F. Peherstorfer, A special class of polynomials orthogonal on the unit circle including the associated polynomials, Constr. Approx. 12 (1996) 161-185.

[23] W.T. Reid, Ordinary Differential Equations, Wiley, New York, 1971.

[24] W. Rudin, Real and Complex Analysis, 3rd Edition, McGraw-Hill, New York, 1987.

[25] B. Simon, Functional Integration and Quantum Physics, in: Pure and Applied Mathematics, Vol. 86, Academic Press, New York, 1979.

[26] B. Simon, Kotani theory for one dimensional stochastic Jacobi matrices, Comm. Math. Phys. 89 (1983) $227-234$.

[27] B. Simon, A new approach to inverse spectral theory, I. Fundamental formalism, Ann. Math. 150 (2) (1999) 1029-1057.

[28] B. Simon, A canonical factorization for meromorphic Herglotz functions on the unit disk and sum rules for Jacobi matrices, J. Funct. Anal. to appear.

[29] B. Simon, Orthogonal Polynomials on the Unit Circle, AMS Colloquium Publications Series, expected 2004.

[30] B. Simon, A. Zlatoš, Sum rules and the Szegö condition for orthogonal polynomials on the real line, Comm. Math. Phys. 242 (2003) 393-423. 
[31] T. Stieltjes, Recherches sur les fractions continues, Anns. Fac. Sci. Univ. Toulouse 8 (1894-1895) J1-J122;

T. Stieltjes, Anns. Fac. Sci. Univ. Toulouse 9, A5-A47.

[32] G. Szegő, Orthogonal Polynomials, Amer. Math. Soc. Colloq. Publ., 3rd Edition, Vol. 23, American Mathematical Society, Providence, RI, 1939, 1967.

[33] E.C. Titchmarsh, Eigenfunction Expansions Associated with Second-Order Differential Equations, Part 1, 2nd Edition, Clarendon Press, Oxford, 1962.

[34] S. Verblunsky, On positive harmonic functions: a contribution to the algebra of Fourier series, Proc. London Math. Soc. 38 (1935) 125-157.

[35] S. Verblunsky, On positive harmonic functions (second part), Proc. London Math. Soc. 40 (1936) 290-320. 